15

\section{THE EFFECTS OF}

I. Z. Lien, E Dropcho, L Mead, D A. Ingram, and L. S. Haneline, Department of Pediatrics, Indiana Purpool of Medicine, Indianapolis, IN.

Purpose of Study: Diabetes Mellitus (DM) is the most prevalent risk factor for acquiring vascula disease. Since optimal glycemic control delays onset and progression of vascular morbidities, hyperglycemia is hypothesized to be a key pathogenic factor for vasculopathies. Vascular integrity require endothelial repair and angiogenesis. Recently, circulating endothelial progenitor cells (EPCs) were shown to have a critical role in promoting endothelial repair and angiogenesis. Given that infants expose that hyperglycemia alters neonatal EPC function. Thus, our aims were to examine the effect of hyperglycemia on newborn EPC clonogenic capacity, proliferative potential, apoptosis, senescence, and capillary tube formation. Methods Used: EPCs were isolated from multiple cord blood donors a described (Ingram et al, Blood, 104:2752, 2004) and subjected to euglycemia or hyperglycemia (10-100 $\mathrm{mM}$ dextrose) before conducting cellular and functional assays. Limiting dilution assays assessed EPC colony formation. Single cell assays quantitated the proportion of single EPCs capable of dividing. Proliferation potential was also assessed by population doubling studies. Apoptosis was evaluated using a TUNEL assay while senescence was examined using an acidic $\beta$-galactosidase method. Matrigel assays assessed capillary tube forming ability. Summary of Results: Hyperglycemia $(10-100 \mathrm{mM})$ resulted in reduced EPC colony formation compared to controls $(n=3, p<0.05)$. Hyperglycemia led to decreased EPC proliferative potential as reflected by a decrease in the number of single EPC cells dividing $(n=96$ single cells) and a lower cumulative population doubling level. In addition, increased apoptosis was detected after a 24 or 48 hour exposure to high dextrose concentrations $(50 \mathrm{mM}$ and $100 \mathrm{mM}$ ) compare to controls $(n=3, p<0.003)$. Senescence assays are underway, and preliminary matrigel assays sugges that hyperglycemia treated EPCs exhibit reduced capillary tube formation. Conclusion: These dat demonstrate that neonatal EPCs have decreased clonogenic potential after hyperglycemia treatment. The mechanisms for the observed decrease in clonogenic capacity may include an increase in both apoptosis and senescence together with a decrease in proliferation. Collectively, these observations may have
implications for the pathogenesis of vascular disease in diabetic patients as well as in infants exposed to implications for the pathogenesis of

16

MYOCARDIAL EXPRESSION OF MUTANT P53 PROTECTS AGAINST DOXORUBICIN-INDUCED CARDIOMYOCYTE APOPTOSIS AND PRESERVES HEART FUNCTION.

W Zhu, LJ Field, Herman B Wells Center for Pediatric Research, Department of Pediatrics, Indiana University School of Medicine, Indianapolis, IN.

Doxorubicin, a topoisomerase II inhibitor that is commonly used to treat childhood cancer, induces p53-mediated cardiomyocyte apoptosis which can ultimately lead to heart failure. We hypothesized that cardiac-restricted expression of a dominant interfering p53 mutant (CB7) would protect cardiomyocytes from doxorubicin-induced apoptosis in vivo. Age-matched CB7 transgenic mice and control nontransgenic (NTG) mice were treated with a total of $20 \mathrm{mg} / \mathrm{kg}$ of doxorubicin (2 intraperitoneal injection of $10 \mathrm{mg} / \mathrm{kg}$ at 3-day interval). The prevalence of cardiomyocyte apoptosis was assessed via anti-activated caspase-3 immune reactivity and also via terminal deoxynucleotidyl transferase (TdT)-mediated dUTP nick end labeling (TUNEL) staining. Cleavage of poly (ADP-ribose) polymerase (PARP, a substrate of caspase-3) and the level of the anti-apoptotic protein Bcl-xL were also monitored. Cardiac function wa assessed using the two dimensional echocardiography. Our results show that the prevalence of cardiomyocyte apoptosis was reduced in CB7 mice as compared to NTG mice following doxorubicin injectio (activated caspase 3 immune reactivity: $0.85 \pm 0.14$ positive cardiomyocytes $/ \mathrm{mm}^{2}$ vs. $1.98 \pm 0.24$ positive cardiomyocytes $/ \mathrm{mm}^{2}, \mathrm{p}<0.05, \mathrm{n}=6$; TUNEL staining: $0.045 \pm 0.006 \%$ of total cardiomyocyte nuclei vs $0.146 \pm 0.009 \%, \mathrm{p}<0.01, \mathrm{n}=5$ ). In agreement with this, caspase-dependent PARP cleavage was not
detected in the doxorubicin-treated $\mathrm{CB} 7$ hearts but was detected in the doxorubicin-treated NTG. Bcl-xL expression was induced to a greater extent in CB7 mice as compared to NTG mice. Finally, fractional
shortening was better preserved in the CB7 mice as compared to the NTG mice $(7.79 \pm 1.35 \%$ reduction shorion post-injectin $\backslash 5.28 .87 \pm 5.48 \%, p<0.05$, no observed in DOX treated CB7 mice appeared to exceed that which would be expected based on the dex expression of $\mathrm{CB} 7$ protects against doxorubicin-induced cardiomyocyte apoptosis and preserves heart function. The anti-apoptotic effects function suggests that antagonization of p53 may engender additional cardioprotective activity.

\section{7}

EFFECTS OF PROLONGED STORAGE OR PASTEURIZATION ON THE LEVELS AND ACTIVITY OF HOST DEFENSE PROTEINS IN HUMAN MILK.

K. Zimmerly, R. Clark, Y. Ma, C. Auer, H. Akinbi, Cincinnati Children's Hospital Medical Center and The University Hospital, Cincinnati, $\mathrm{OH}$.

Background: Human milk is the preferred nutrition for neonates due to the multiple nutritional and immunologic benefits over formula. Preterm neonates are often fed human milk that has been stored frozen for up to 3 months. Human milk is sometimes pasteurized prior to feeding to preterm neonates to reduce the transmission of pathogens. Goal: To assess changes in the concentrations and activity of host defense proteins in human milk following prolonged storage or pasteurization. Methodology: (A) Samples obtained through Cincinnati Children's Hospital Human Milk Bank were frozen at $-20{ }^{\circ} \mathrm{C}$ fo 2 days, 4,8 , or 12 weeks prior to analysis. Whey fraction was isolated and protein analyzed by dot blo protein analysis followed by immunoreaction with antibodies to human lysozyme, lactoferrin, lactoperoxidase, secretory leukoprotease inhibitor, HNP 3 , or human beta defensin 2 . Protein levels were (B) Freshly-collected human milk samples were pasteurized at $65^{\circ} \mathrm{C}$ for 30 minutes, then stored at -20 ${ }^{\circ} \mathrm{C}$ and analyzed by dot blot for host defense proteins as described in $\mathrm{A}$. (C) Muramidase activity was assessed by incubating $10 \mu \mathrm{g}$ total protein with a suspension of Micrococcus luteus and calculated from the rate of decrease in optic density. Recombinant human lysozyme was used to generate a standar curve. Results: Levels of lysozyme, lactoferrin, and lactoperoxidase were not significantly changed fo up to 8 weeks of storage at $-20^{\circ} \mathrm{C}$. In contrast, levels of sIgA were significantly reduced within 1 week at $-20{ }^{\circ} \mathrm{C}(40 \%$ at 1 week, $10 \%$ at 2 weeks, and $<5 \%$ at 8 weeks $\mathrm{p}<0.05$ relative to fresh milk). Muramidase also activity decreased sharply with storage $(120$ units $/ \mu \mathrm{g}$ vs. 260 units, $\mathrm{p}<0.02)$. In comparison with age-matched control samples, concentrations of lysozyme, lactoferrin, lactoperoxidase, $\mathrm{sIgA}$, and muramidase activity were unaltered following pasteurization. HNP-3 and human beta defensin 2 were not detected. Discussion: Although the nutritional value of human milk may be preserved following prolonged storage at $-20{ }^{\circ} \mathrm{C}$, protein activity may be significantly compromised thereby
reducing the immunologic benefits from human milk. Pasteurization of milk to reduce transmission of viruses appears safe with respect to levels and activity of host defense proteins.

\section{8}

CIRCULATION PLAYS AN ESSENTAL ROLE IN DISTRIBUTING HEMATOPOIETIC PROGENITORS FROM THE YOLK SAC TO THE EMBRYO PROPER; LESSONS FROM THE NCX1-NULL MOUSE.

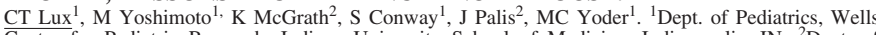
Center for Pediatric Research, Indiana University School of Medicine, Indianapolis, IN; ${ }^{2}$ Dept. of Pediatrics, Center for Pediatric Biomedical Research, University of Rochester School of Medicine,
Rochester, NY. The yolk sac is the lone site of primitive hematopoiesis. However, the role of the yolk sac in generating definitive hematopoietic progenitors has remained controversial since the onset of circulation alters the localization of hematopoietic progenitors. The Ncxi knockon mouse falls to initiate a heartbeat and thus in an embryonic environment lacking circulation. Embryos were harvested from timed Ncx1 heterozyin an embryonic environment lacking circulation. Embryos were harvested from timed Ncx1 heterozy-
gote crosses beginning at the onset of circulation at embryonic day 8.5 (E8.5) and ending 36 hours late gote crosses beginning at the onset of circulation at embryonic day 8.5 (E8.5) and ending 36 hours late
(E10). The developmental stage of each embryo was determined by counting somite pairs. All embryo (E10). The developmental stage of each embryo was determined by counting somite pairs. All embryos from the embryo proper. The tissues were digested and plated in methylcellulose. Colonies were counted after seven days. At E8.5, definitive hematopoietic progenitors are enriched in the YS more than 28 -fold compared to the PSp in both wild type and Ncx1-null embryos. This ratio drops to 3 -fold enrichment in the YS following progenitor redistribution by a functional circulation (E10) in wild type embryos, as reported previously (McGrath, et al. Blood 2003). The YS of Ncx1-null embryos, which lack a functional circulation, contain as many definitive progenitors as wild type YS and PSp combined. Few hematopoietic progenitors are found in the Ncx1-null PSp as late as E10, resulting in a 72-fold enrichment of progenitors in the Ncx1-null YS. We conclude that the PSp does not appear to function as a site of active hematopoiesis prior to E10. Furthermore, our findings support a model in which large numbers of
definitive hematopoietic progenitors are generated in the YS between E8.5 and E10 and are redistributed to the embryo proper of the mouse embryo once cardiac function initiates a functional circulation.

\section{9}

DEFICIENCY IN GATA-4 OR GATA-6 DIMINISHES DEFINITIVE HEMATOPOIESIS IN MURINE EMBRYONIC STEM CELL DERIVED EMBRYOID BODIES.

M Pierre and M Yoder, Department of Pediatrics, Indiana University School of Medicine, Indianapolis,

Formation of mesoderm derived blood islands in the mouse embryonic yolk sac requires the presence
of visceral endoderm (VE) and VE derived factors. Murine embryonic stem (ES) cells can be differenof visceral endoderm (VE) and VE derived factors. Murine embryonic stem (ES) cells can be differentiated into embryoid bodies (EBs) which serve as an in vitro model recapitulating many embryonic developmental processes, including formation of early hematopoietic cells. Previous investigators have reported that differentiation of ES cells deficient in either GATA-4 or GATA-6 results in EBs with disrupted differentiation of visceral endoderm and defective blood island formation. In the current study, we have compared GATA- 4 and GATA-6 null ES cell derived EBs to wild-type EBs in their ability to
commit to early hematopoietic lineages using hematopoietic progenitor colony assays, and used RT-PCR commit to early hematopoietic lineages using hematopoietic progenitor colony assays, and used RT-PCR to assess the expression of endoderm genes. As expected, we observed differences in expression of
endoderm genes in wild-type and GATA-4 or GATA-6 null EBs. Blast colony forming cell assays from day 2 and day 3 EBs revealed no difference between the ability of wild-type and GATA-4 or GATAnull EBs to differentiate into hemangioblasts. Analysis of primitive erythroid progenitors from day 4,5 6 and 7 wild-type and homozygous deficient EBs also showed similar numbers of primitive erythroid 9 progenitor colonies. In contrast, comparisons of definitive hematopoietic progenitor colonies from day 8, (p-values $<0.05$ ) compared to wild-type. Strikingly, definitive progenitor colony numbers are rescued (p-values $<0.05$ ) compared to wild-type. Strikingly, definitive progenitor colony numbers are rescued nearly to wild-type levels after the addition of visceral endoderm derived factors during EB differentinot necessary for hemangioblast, primitive erythroid, or definitive progenitor emergence from EBs but play a role in definitive progenitor expansion in EBs. 